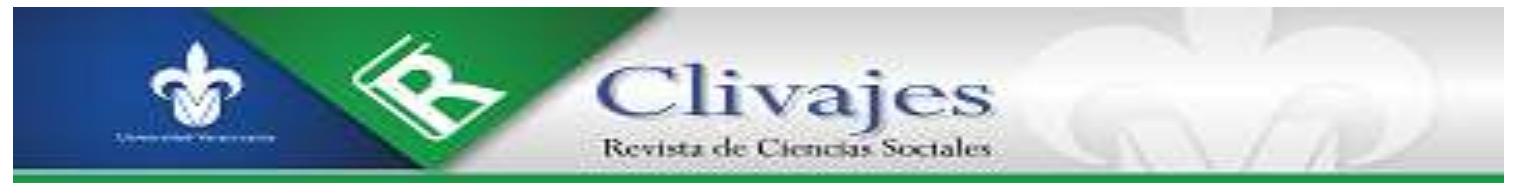

Iliana Yarethzi Viramontes Márquez

yayaviramontes@gmail.com

Oficina del Alto Comisionado de las Naciones Unidas para los Refugiados en Guadalajara

Universidad de Guadalajara

JALISCO, UN ESTADO MIGRANTE. ANUARIO DE MIGRACIÓN DE ORIGEN, DESTINO, TRÁNSITO Y RETORNO

EN JALISCO 2018. ANÁLISIS, CRÍTICAS Y PROPUESTAS

DOI: https://doi.org/10.25009/clivajesrcs.i15.2690

Clivajes. Revista de Ciencias Sociales. Año VIII, número 15, enero-junio 2021, pp. 37-57.

https://clivajes.uv.mx/index.php/Clivajes

Instituto de Investigaciones Histórico-Sociales, Universidad Veracruzana

Clivajes. Revista de Ciencias Sociales/ISSN: 2395-9495/IIH-S, UV/Xalapa, Veracruz, México

Recibido: 24/03/2021

Aceptado: 29/04/2021

Dictaminado: 10/05/2021

(c) (i) ()

Clivajes. Revista de Ciencias Sociales (ISSN: 2395-9495), Año VIII, Núm. 15, enero-junio, 2021 Instituto de Investigaciones Histórico-Sociales, Universidad Veracruzana, México 


\title{
JALISCO, Un ESTADO MigRANTE. ANUARIO DE MIGRACIÓN DE ORIGEN, DESTINO, TRÁNSITO Y RETORNO EN JALISCO 2018. ANÁLISIS, CRÍTICAS Y PROPUESTAS
}

\author{
Iliana Yarethzi Viramontes Márquez ${ }^{*}$
}

\begin{abstract}
Resumen
En los últimos años, la acción de los gobiernos locales en las relaciones internacionales ha cobrado gran importancia; sin embargo, poco se ha abonado al estudio de la paradiplomacia desde una perspectiva práctica y centrada en un fenómeno o una realidad en particular, como la migración. Por ello, en este ensayo se analiza la elaboración del Anuario de migración de Jalisco, así como su importancia para los gobiernos y actores locales interesados en comprender la migración a escala local e internacional.
\end{abstract}

Palabras clave: Migración, Flujos migratorios, Gobiernos locales, Actores locales, Paradiplomacia

JALISCO A MIGRANT STATE. YEARBOOK OF MIGRATION OF ORIGIN, DESTINATION, TRANSIT AND RETURN IN JALISCO 2018. ANALYSIS, CRITICISM AND PROPOSALS

\begin{abstract}
In recent years, the action of local governments in international relations has gained great importance; however, little has been done in the study of paradiplomacy from a practical perspective and focused on a particular phenomenon or reality, such as migration. For this reason, this essay analyzes the development of the Jalisco Migration Yearbook, as well as its importance for local governments and actors interested in understanding migration at a local and international levels.
\end{abstract}

Keywords: Migration, Migratory flows, Local governments, Local actors, Paradiplomacy

\section{JALISCO Un ÉtAT MIGRANT. ANNUAIRE DE MIGRATION D’ ORIGINE, DESTIN, TRANSIT ET RETOUR À JALISCO 2018. ANALYSE, CRITIQUES ET PROPOSITIONS}

\section{Résumé}

Ces derniers temps, l'action des gouvernements locaux dans les relations internationales a pris de l'importance ; cependant, on a peu abondé sur l'étude de la para-diplomatie à partir d'une perspective pratique et centrée dans un phénomène ou une réalité en particulier comme la migration. C'est pourquoi, dans cet essai on analyse l'élaboration de l'Annuaire de migration de Jalisco, de même que

* Asociada de Protección en la Oficina del Alto Comisionado de las Naciones Unidas para los Refugiados en Guadalajara y profesora asistente de Redes Públicas Internacionales en la licenciatura de Relaciones Internacionales de la Universidad de Guadalajara, de la cual es egresada. Ha sido encuestadora, asistente de investigación y coordinadora de Trabajo de Campo en el Mexican Migration Project (MMP), auspiciado por las universidades de Guadalajara y Princeton (2016 -2018); también, coordinadora de Derechos Humanos y Desarrollo Integral, así como del área de investigación del Instituto Jalisciense para Migrantes. 
son importance pour les gouvernements et acteurs locaux intéressés à comprendre la migration à l'échelle locale et internationale.

Mots clés : Migration, Flux migratoires, Gouvernements locaux, Acteurs locaux, Para-diplomatie

\section{INTRODUCCIÓN}

¿Qué es la migración? Para definirla no basta recorrer las vías del tren, el muro fronterizo o las costas del mediterráneo. No, la verdad es que la migración es una condición inherente al ser humano desde que existe como especie, moviéndose constantemente de un lugar a otro: ni la agricultura, ni los asentamientos, ni el Estado le han quitado lo nómada. La movilidad humana no depende de su condición socioeconómica, racial o cultural. Migran los pobres y los ricos, las minorías y las mayorías. En realidad, la migración es lo que les ha pasado a muchas familias. Lo que es cierto, es que hay ciertos grupos de personas para los que migrar es no sólo instintivo, sino un asunto de supervivencia. Los desplazamientos forzados y, en general, las crisis económicas, sociales y políticas del orbe son el lado oscuro del gran proceso de la interculturalidad.

Si migrar es salir de los lugares de residencia para asentarse en un contexto distinto, será lógico concluir que todos los aspectos de su nueva vida se verán afectados. Es por ello que en este ensayo se sostiene que la movilidad humana se debe estudiar desde la transversalidad de los derechos, que además afecta no sólo a quienes se van, sino a quienes dejan, a quienes conocerán y, en algunos casos, a personas que nunca conocerá, pero que vivirán los efectos de sus antepasados migrantes.

Así es como el fenómeno de la migración se ha visto inmerso tanto en las dinámicas del sistema mundo actual, como en las estrategias propuestas por distintos Organismos Internacionales que en los últimos años han desarrollado una perspectiva local para el análisis de distintos fenómenos, entre ellos, el migratorio. Esto ha evidenciado la necesidad de contar con información y datos desagregados como insumo para diseñar e implementar programas y políticas públicas concentradas en atender problemáticas de las poblaciones migrantes.

En este contexto, en el presente ensayo se analiza (desde una perspectiva crítica y propositiva en torno a la generación de datos estadísticos migratorios desde los gobiernos y actores locales) el proceso de elaboración del primer anuario de migración en Jalisco. El análisis se acompaña de críticas y propuestas, resultado de la experiencia 
acumulada en el Instituto Jalisciense para los Migrantes (IJAMI). En consonancia con lo anterior, el objetivo principal de este escrito es aportar elementos para el estudio e intervención de la migración desde la perspectiva de las Relaciones Internacionales y la paradiplomacia.

Para ofrecer una lectura organizada, clara y amena, el texto se estructura en cinco apartados: en el primero, se explican las funciones que tuvo el IJAMI en la administración pública; en el segundo, se aborda el proceso de integración del anuario de migración (sus etapas de elaboración, las acciones realizadas y la metodología utilizada en cada una de ellas); en el tercer apartado, se presenta un análisis en torno a la generación de datos estadísticos desde la perspectiva de las Relaciones Internacionales, el ámbito gubernamental y temas migratorios; en el cuarto, se plantea una serie de reflexiones, criticas y apuntes técnicos en torno a la paradiplomacia, utilizando el anuario como ejemplo práctico; en el quinto apartado, se realiza un análisis de los desafíos de la institucionalidad local sobre la migración y la carencia de fuentes de información y, finalmente, se presenta las conclusiones.

EL Instituto JaLisciense PARA LOS Migrantes (IJAmi) Y SU IMPACTO EN LA ADMINISTRACIÓN PÚBLICA

Durante la administración 2013-2018 y por medio de decretos, el gobierno estatal de Jalisco creó distintos órganos públicos desconcentrados con el propósito de atender a las poblaciones consideradas "vulnerables" en el Plan Estatal de Desarrollo; entre ellos, se creó el Instituto de la Mujer, el Instituto de la Juventud y el Instituto Jalisciense para los Migrantes (IJAMI), el último de los cuales, subordinado a la Secretaría de Desarrollo e Integración Social (SEDIS) del Gobierno del estado de Jalisco, tenía por objeto ejecutar las líneas de acción tendientes a alcanzar los objetivos establecidos en el Plan Estatal de Desarrollo en materia de migración (Secretaría General de Gobierno, 2015). 


\section{Cuadro 1.}

\section{Organigrama del Instituto Jalisciense para los Migrantes ${ }^{1}$}

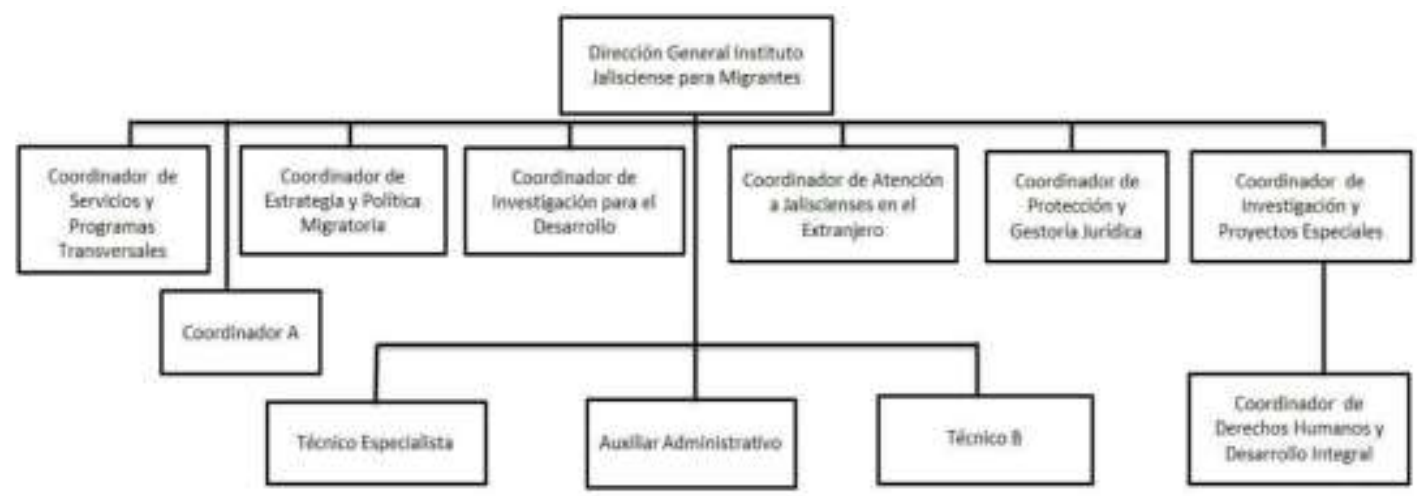

Fuente: Elaboración propia con base en el organigrama realizado para el Manual de Operaciones y Procedimientos del Instituto Jalisciense para los Migrantes, 2018.

En este contexto, bajo la Coordinación de Derechos Humanos y Desarrollo Integral se elaboró el anuario Jalisco, un Estado migrante, en colaboración con el Instituto de Información Estadística y Geográfica de Jalisco, y FM4 Paso Libre. Resulta importante exponer esto, porque en ese momento Jalisco era (junto con Puebla, Guanajuato y Michoacán) una de las pocas entidades federativas que contaba con una dependencia estatal exclusiva para la atención del fenómeno migratorio, y que no sólo se concentraba en la atención directa de personas, sino que también tenía un enfoque de estudio sobre dicho fenómeno, lo cual se refleja en la elaboración de reportes de investigación del instituto y trabajos como el anuario.

JALISCO, UN ESTADO MIGRANTE. ANUARIO DE MIGRACIÓN DE ORIGEN, DESTINO, TRÁNSITO Y RETORNO EN JALISCO 2018

De acuerdo con la Organización Internacional para las Migraciones (OIM), el gran tema de nuestra era es precisamente la migración. Es un fenómeno que se convierte en problema por la definición de las fronteras y el movimiento de las personas a través de ellas, lo cual nos remite a conceptos clave de las Relaciones Internacionales, como Estado-Nación. El mundo ha cambiado y con él la concepción del Estado-Nación, el Leviatán no puede ver ni controlarlo todo; de ahí la necesidad de tener y conocer los

${ }^{1}$ El organigrama presenta un error en la jerarquía de la Coordinación de Derechos Humanos y Desarrollo Integral, que dependía directamente de la Dirección General del Instituto Jalisciense para los Migrantes. 
datos estadísticos del tema migratorio en Jalisco y sus municipios, y de que se priorice la generación de conocimiento, la comprensión de datos, así como el papel significativo de los gobiernos locales en la producción y manejo de datos estadísticos, y específicamente en el tema migratorio, la importancia de saber qué datos es posible desagregar a nivel de municipio y/o regiones administrativas.

Si bien esto se ha venido manejando en la agenda internacional, principalmente a partir del la firma del Pacto Mundial para la Migración Ordenada, Segura y Regular, la realidad es que difícilmente se puede obtener información estadística, desagregada a nivel municipal, de los distintos flujos migratorios, por lo que el desarrollo del anuario se basó principalmente en la compilación de datos ya existentes, con el fin de realizar un mapeo de la información con la que sí se contaba, es decir, se elaboró un estado de la cuestión de los datos estadísticos migratorios de Jalisco.

A continuación se analiza la metodología utilizada para desarrollar el trabajo titulado Jalisco un estado migrante. Anuario de migración de origen, destino, tránsito y retorno en Jalisco 2018.

\section{Metodología}

En el artículo titulado "Coordenadas metodológicas. Dé cómo armar el rompecabezas", Jorge Durand (2014) establece cuatro coordenadas metodológicas necesarias para delimitar una investigación, de forma tal que se aprecie la dimensión y el formato general que ésta tendrá. El proyecto del anuario se llevó a cabo con base en tres de las cuatro coordenadas metodológicas propuestas por Durand: la temporal, la territorial y la temática; debido a la naturaleza del trabajo, la coordenada teórica no fue utilizada.

La coordenada territorial se refiere al lugar en el tendrá lugar la investigación. Para la elaboración del anuario ésta se determinó desde el inicio. Cabe mencionar que, en el capítulo 1 y en el capítulo 3, esta coordenada fue más específica, ya que se contemplaron las 12 regiones administrativas de Jalisco. Se hizo de esa manera porque resultaba más sencillo contemplar 12 regiones que 125 municipios.

La coordenada temporal es el periodo para estudiar; el anuario tiene una temporalidad de 2012 a 2017. Esta coordenada se contempló así por la administración durante la cual se realizó la publicación; sólo el capítulo 1 tiene un cambio, de 2012 a 2016, por la carencia de información sobre remesas para el año 2017. 
La coordenada temática se refiere al tema específico que se piensa investigar, en este caso, migración internacional. Cabe mencionar que en términos conceptuales se puede hacer la observación de que se habla de migración internacional y posteriormente definir por flujos migratorios, pero es importante considerar que el anuario es un trabajo de recopilación de datos y no de investigación en términos académico-teóricos, principalmente para el capítulo 6, ya que el refugio es una condición y no un flujo migratorio; no obstante, se tomaron decisiones funcionales porque, en términos prácticos, Jalisco es una entidad federativa en donde confluyen los cuatro flujos migratorios y así se plasmó en el anuario.

Para el caso particular del anuario se tienen dos categorías de coordenadas metodológicas; la primera es la general, en ésta se encuadra el anuario en su totalidad, como se muestra en el diagrama 1; y la segunda categoría es la particular, en la que se delimita cada capítulo y la única diferencia entre cada uno es el sujeto de estudio; en el diagrama 2 se muestran las coordenadas metodológicas por capitulo.

\section{Cuadro 2.}

Diagrama de las coordenadas metodológicas generales del anuario de migración

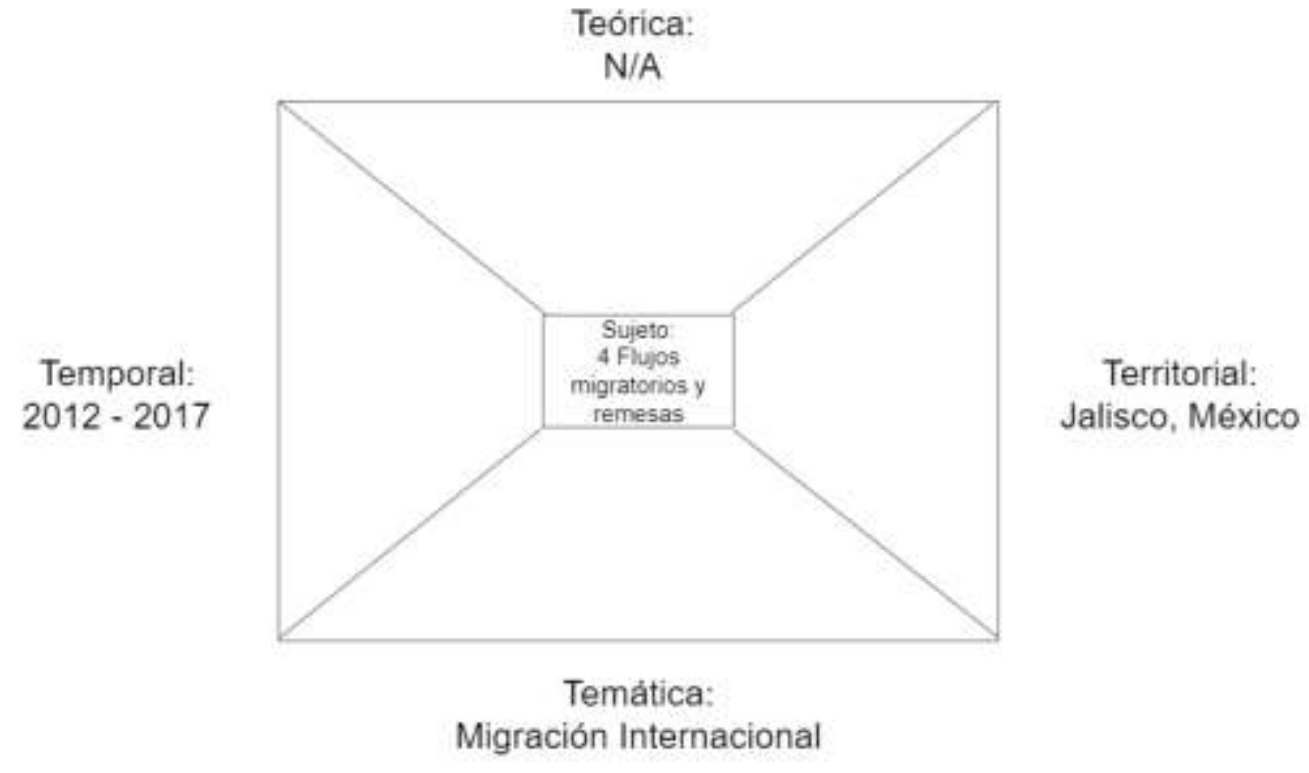

Fuente: Elaboración propia con base en Durand (2014). 


\section{Cuadro 3.}

Diagrama de las coordenadas metodológicas particulares del anuario de migración

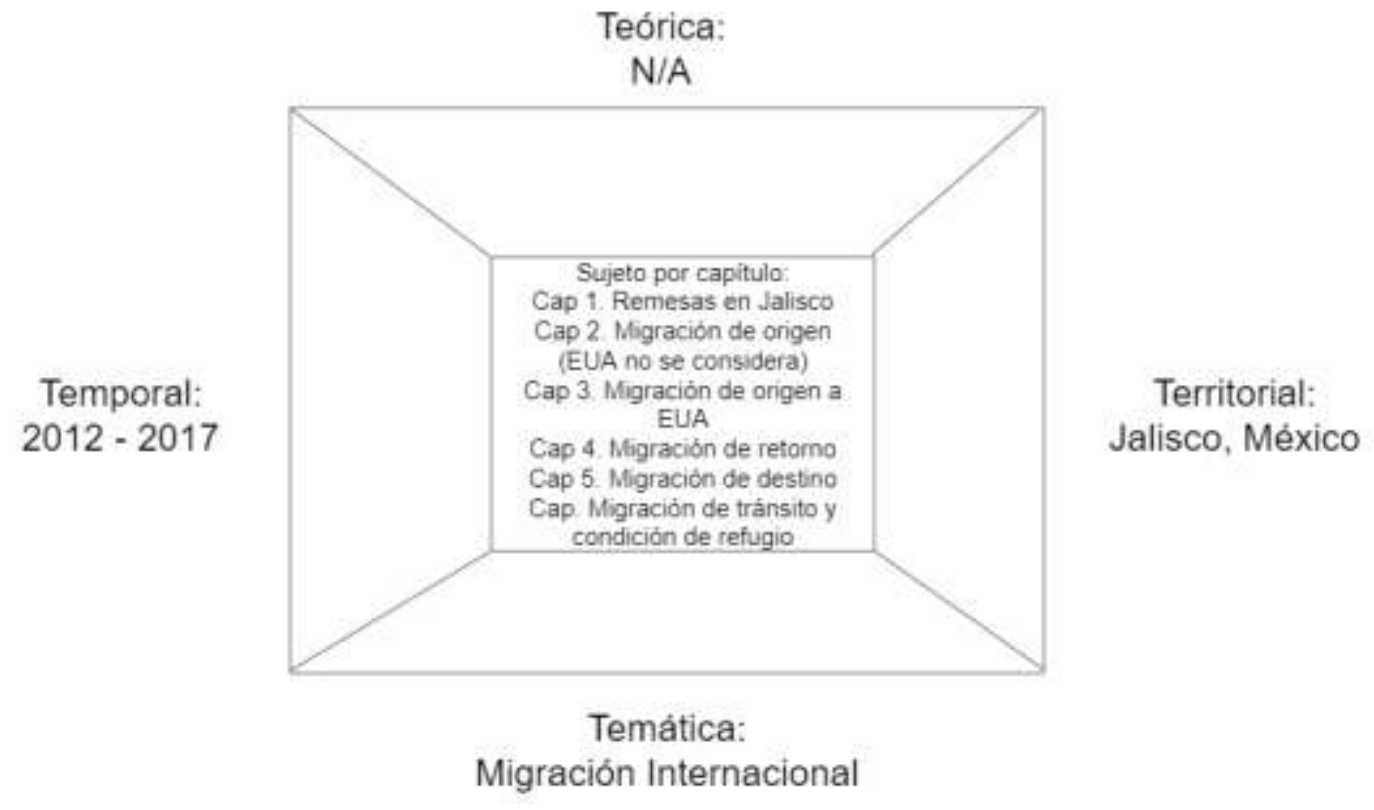

Fuente: Elaboración propia con base en Durand (2014).

Etapas de elaboración

En términos de investigación, el primer paso fue forjar un Estado del Arte de anuarios, con el fin de saber cuál sería la ruta que seguiría el anuario titulado Jalisco, un Estado migrante. Anuario de migración de origen, destino, tránsito y retorno en Jalisco 2018, y conocer trabajos previos. Es importante mencionar que el proyecto no pretendía encontrar el hilo negro de la migración en Jalisco, sino hacer una compilación de datos estadísticos en torno al tema migratorio, y que por primera vez esta entidad federativa contara con un ejercicio como éste.

El documento fue realizado bajo un modelo muy similar a los Anuarios de Migración y Remesas que año con año editan la Fundación BBVA y el Consejo Nacional de Población (CONAPO). Una vez que se determinó que estos serían el ejemplo a seguir, se hizo una revisión profunda de cada uno de los anuarios publicados durante los últimos años; en particular se revisaron los correspondientes a los años 2014 a 2017. Esta labor de investigación permitió conocer las principales fuentes estadísticas, así como los datos 
que se encuentran desagregados a nivel estatal y municipal para poder determinar la lista de temas finales.

Fue por medio de este proceso de investigación que se definieron cuáles serían las fuentes de información para cada capítulo del anuario. Cabe mencionar que todas son instituciones de gobierno, salvo en el capítulo 6, en la sección de migración de tránsito y refugio en el que participó la organización social FM4 Paso Libre. El texto consta de seis capítulos:

1. Remesas en Jalisco: Banco de México, por medio de la información de remesas.

2. Jaliscienses en el mundo: Instituto de Mexicanos en el Exterior, por medio de las matrículas consulares expedidas.

3. Jaliscienses en Estados Unidos: Instituto de Mexicanos en el Exterior, por medio de las matrículas consulares expedidas.

4. Repatriación a Jalisco: Instituto Nacional de Migración, por medio de los boletines estadísticos, cuadros de repatriación de mexicanos.

5. Inmigración Internacional a Jalisco: Instituto Nacional de Migración, por medio de los boletines estadísticos, cuadros de documentación y estancia en México, y cuadros de extranjeros presentados y devueltos.

6. Migración de Tránsito y Refugio: FM4 Paso Libre para migración de tránsito, y la Comisión Mexicana de Ayuda a Refugiados para solicitudes de refugio en Jalisco.

Cabe destacar que en la elaboración de estos capítulos, se colaboró con otros actores locales que trabajan temas de migración y/o estadística. Por ejemplo, como se mencionó en párrafos precedentes, para temas propios de migración, y muy específicamente de migración de tránsito, se colaboró con FM4 Paso Libre, que es una organización sin fines de lucro dedicada a la defensa y promoción de derechos humanos de las personas migrantes y refugiadas mediante la atención humanitaria integral, la incidencia en políticas y la investigación desde el occidente de México (FM4 Paso libre, s/f), que forma parte de la Red de Organizaciones Defensoras de Migrantes (REDODEM) y es uno de los socios del Alto Comisionado de las Naciones Unidas para los Refugiados en México (ACNUR) en la implementación del Programa de Integración Local (PIL).

Respecto a temas estadísticos y elaboración de cartografía, se trabajó con el Instituto de Información Estadística y Geográfica del estado de Jalisco (IIEG), organismo encargado de realizar estudios acerca de diferentes temas, desde geográficos y 
sociodemográficos, hasta económicos, e integrar boletines mensuales, entre otros materiales.

Las fuentes de información para construir la estadística fueron principalmente instituciones de gobierno, por lo que dependiendo de la institución, la manera de obtener los datos cambiaba. Toda la información fue obtenida de dos formas; en la primera se descargaron los archivos directamente de la página de internet, lo que resultaba sencillo y sólo era necesario guardar orden en el título de los archivos y/o carpetas, y en la segunda, por medio de solicitudes de transparencia, lo cual retrasaba la obtención de archivos, además de que los formatos de las respuestas de transparencia eran poco amigables para trabajarlos, ya que se enviaban oficios escaneados. En la tabla 1 se detalla, por institución, la forma en la que se obtuvieron los datos, su formato y extensión, así como observaciones.

Tabla 1. Mecanismos de obtención de datos según la institución

\begin{tabular}{|c|c|c|c|}
\hline Institución & $\begin{array}{l}\text { Mecanismo de obtención de } \\
\text { información }\end{array}$ & Formato y extensión & Obsevaciones \\
\hline $\begin{array}{l}\text { Banco de } \\
\text { México }\end{array}$ & $\begin{array}{l}\text { Página de Internet, Sistema de } \\
\text { información Económica, Balanza } \\
\text { de pagos, Remesas }\end{array}$ & Tablas .XML & $\begin{array}{l}\text { La organización y distribución de } \\
\text { las tablas facilita el trabajo; el único } \\
\text { apunte aquí es que el reporte de } \\
\text { remesas es trimestral por lo que se } \\
\text { tuvo que hacer la modificación al } \\
\text { archivo y sacar el dato anual. }\end{array}$ \\
\hline $\begin{array}{l}\text { Instituto de } \\
\text { Mexicanos en } \\
\text { el Exterior }\end{array}$ & $\begin{array}{l}\text { (1) Información a nivel general: } \\
\text { página de internet } \\
\text { (2) Información desagregada: } \\
\text { solitud de trasparencia }\end{array}$ & $\begin{array}{ll}\text { (1) Tablas .XML } \\
\text { (2) Links para mapas } \\
\text { interactivas .PDF, ya que } \\
\text { era una contestación de } \\
\text { trasparencia por correo } \\
\text { electrónico }\end{array}$ & $\begin{array}{l}\text { (1) Tablas de Excel, organizadas } \\
\text { de manera muy sencilla, lo } \\
\text { que facilitaba su manejo. } \\
\text { (2) Muy complicados para } \\
\text { manejarse; se requería } \\
\text { navegador especial, versiones } \\
\text { específicas de complementos; } \\
\text { tomaba mucho tiempo } \\
\text { completar la información y } \\
\text { aumentaba la probabilidad de } \\
\text { errores. }\end{array}$ \\
\hline $\begin{array}{l}\text { Instituto } \\
\text { Nacional de } \\
\text { Migración }\end{array}$ & $\begin{array}{l}\text { Página de internet, Boletines } \\
\text { estadísticos }\end{array}$ & Tablas .XML & $\begin{array}{l}\text { Los boletines estadísticos hacen } \\
\text { muy sencillo encontrar y descargar } \\
\text { los archivos; sin embargo, la } \\
\text { distribución y el acomodo de las } \\
\text { tablas es poco amigable al } \\
\text { momento de descargar los datos a } \\
\text { nivel estatal. }\end{array}$ \\
\hline $\begin{array}{l}\text { Comisión } \\
\text { Mexicana de } \\
\text { Ayuda al } \\
\text { Refugiado }\end{array}$ & Solicitud de trasparencia & $\begin{array}{l}\text { Tablas en extensión .PDF, ya } \\
\text { que era una contestación de } \\
\text { trasparencia escaneadas. }\end{array}$ & $\begin{array}{l}\text { El formato era sumamente } \\
\text { complicado para manejar la } \\
\text { información, se tenía que pasar la } \\
\text { tabla completa de la hoja escaneada } \\
\text { a un documento de Excel. }\end{array}$ \\
\hline
\end{tabular}

Fuente: Elaboración propia. 


\section{REFLEXIONES EN TORNO A LA PARADIPLOMACIA}

En este apartado se invita al análisis y la reflexión sobre la importancia que la acción de los gobiernos y actores locales tienen en la actualización sobre estrategias y proyectos de organismos y organizaciones internacionales para su implementación en los espacios subnacionales, así como el conocimiento de los actores locales que trabajan el tema de interés con el fin de fortalecer las acciones paradiplomáticas.

Al respecto, cabe acotar que, en los últimos años, en el estudio de las Relaciones Internacionales, se ha dado un particular énfasis a lo local (bien lo dijo el pensador Séneca "(...) nuestro universo sería una cosa tan limitada si no ofreciera a cada época algo que investigar"). Y con el surgimiento de conceptos como paradiplomacia y regionalismo, entre otros, se hizo evidente cómo pueden verse reflejados fenómenos internacionales en el ámbito local, como es el caso de la migración. Es en temas como éste que la paradiplomacia cobra mucha pertinencia, ya

que invita al análisis de la incidencia e importancia que adquieren los gobiernos locales en asuntos internacionales, lo cual se asume como un fenómeno de las Relaciones Internacionales contemporáneas que nace en el contexto internacional (Arenas, 2018).

Al respecto, Zidane Zeraoui (2016) aborda la importancia de definir el concepto de paradiplomacia y sus alcances; en ese sentido, habla de la paradiplomacia positiva, la cual refiere actividades de una región, que son complementarias de la diplomacia nacional, y no entran en contradicción con los intereses globales del país. El anuario es ejemplo de este alcance positivo, ya que las acciones del gobierno local estaban alineadas al Plan Nacional de Desarrollo y al Programa Especial de Migración del gobierno federal. El anuario incluso se colocó a la vanguardia, en armonía con las negociaciones promovidas por el Estado mexicano ante las Naciones Unidas en 2018 para la promulgación y firma del Pacto Mundial para una Migración Segura, Ordenada y Regular, sobre el cual se profundizará más adelante.

El anuario expone cómo actualmente el sistema internacional y sus actores, incluyendo a los gobiernos locales, se encuentran inmersos en dinámicas y retos de la globalización; por lo tanto, es una respuesta natural de estos (gobiernos locales) para participar más activamente (Arenas, 2018). Si bien el estado de Jalisco tiene una gran historia de vinculación con otros actores a nivel internacional en una dimensión económica, el anuario significó una apuesta para trabajar en coordinación con otros actores desde el plano social y desde una aproximación técnica. 
Los datos presentados en el anuario son muy importantes porque visibilizan las relaciones que existen entre las personas más allá de lo económico, situaciones y problemáticas que resultan compartidas; ofrece los insumos para darnos cuenta de lo conectados que estamos, de la presencia que tiene Jalisco en el mundo. El anuario es el instrumento por medio del cual exponemos esta interconexión en términos humanos, pensando en aquellos que se fueron y en aquellas personas que están llegando.

Hoy en el paradigma del siglo XXI estamos presenciando la articulación de una nueva gobernanza mundial multinivel que involucra a las ciudades como actores protagónicos en la era de la globalidad al ser espacios de proximidad y laboratorios de innovación desde donde se pueden articular de una mejor forma los actores y las políticas públicas para atender desafíos globales como el cambio climático, las pandemias, los movimientos migratorios, entre otros retos globales, desde perspectivas más cercanas a la ciudadanía (Iglesias, 2020, p. 8).

Esto es sumamente importante, ya que la idea dominante de la migración en Jalisco, desde la academia e instituciones de gobierno, durante mucho tiempo tuvo un enfoque centrado en el flujo migratorio de origen, posteriormente se expusieron el flujo de retorno y las deportaciones. Esto se debió en gran parte a la crisis económica de 2008 y a la imagen de Barack Obama como el "presidente deportador", que dejaba claro el eje principal de trabajo de la agenda migrante, el cual puede verse en los programas sociales que existían en México para atender dicho flujo: Fondo de Apoyo a Migrantes, 3x1, Bienvenido Paisano y Somos Mexicanos, mientras que, en el caso del flujo migratorio de tránsito y la protección internacional, poco se hablaba desde lo institucional, y eran organizaciones como FM4 Paso Libre y El Refugio las que se encargaban de exponer el tema tanto en la esfera académica, como a nivel social y político.

Posteriormente a la llegada de las caravanas migrantes, el gobierno, en sus distintos órdenes, comenzó a tener mayor incidencia en el tema, sobre todo en las entidades federativas del sur del país. En las entidades del centro y norte, el tema migratorio se sigue promoviendo por parte de asociaciones civiles, organismos internacionales, como ACNUR y agencias de cooperación para el desarrollo.

El trabajo de colaboración con diversas instituciones muestra el cambio de enfoque y el fortalecimiento de la red de actores en el tema migratorio, los cuales no se limitan al gobierno, sino que implican a la sociedad civil y nos recuerdan la 
importancia de mantener una red local consolidada que permita que acciones como la integración del anuario se fortalezcan y participen en la esfera internacional. En el diagrama 3 se muestra la red de actores migratorios en Jalisco.

\section{Cuadro 4.}

Diagrama de la Red de actores migratorios en Jalisco 2017

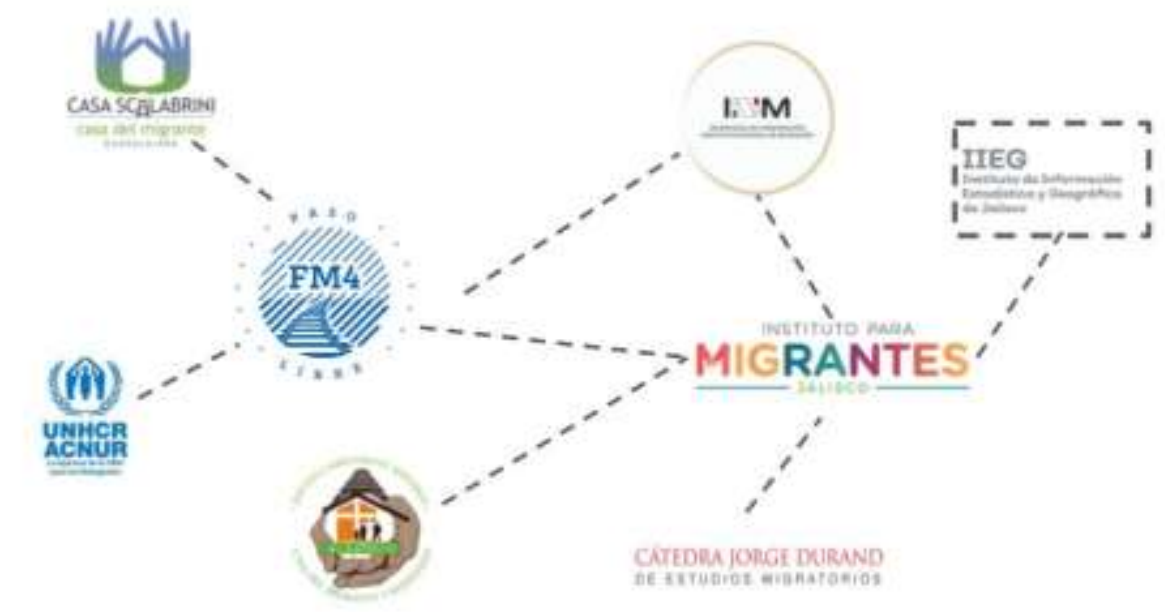

Fuente: Elaboración propia.

Resulta importante señalar que este trabajo fue pensado como un proyecto a largo plazo y que tendría continuidad. En el proyecto se concibió a dicha publicación como la primera de una serie de anuarios por venir. Esto no sucedió así, ¿por qué? Por la falta de continuidad en los proyectos de gobierno. La llegada de una nueva administración gubernamental trae consigo un cambio en la agenda política, es decir, un cambio de prioridades y la revisión de presupuestos. Si bien se tendrán temas, proyectos, políticas públicas y programas a los que se les dará continuidad, no será así con todos.

El anuario forma parte de un tema que actualmente no se encuentra en la agenda de prioridades del gobierno del estado de Jalisco. Aún cuando, en el Plan Estatal de Gobernanza y Desarrollo, el tema migrante se encuentra en el capítulo 6.2 Desarrollo social, en el apartado de grupos prioritarios, la agenda de actividades en torno a ella es muy distinta. Es por ello que resulta vital reconocer que no basta con tener conocimiento técnico sobre un tema, sino que también se hace imperativo que los actores tomadores de decisiones lo tengan en la agenda política. 
La situación actual de la agenda migrante en Jalisco es un claro ejemplo de un caso de estudio en cuanto a lo que implica un cambio de administración. Si bien el IJAMI tenía un gran número de áreas de oportunidad (era un instituto que exponía el tema migrante), hoy en día los principales impulsores de la agenda no son del gobierno local, sino algunos de los actores antes mencionados.

El hecho de que el anuario contemple los cuatro flujos migratorios y las remesas abre la puerta para la colaboración con instituciones locales y con diversos actores del ámbito internacional, tales como el Banco Mundial, la Organización Internacional para las Migraciones, el Alto Comisionado de las Naciones Unidas para los Refugiados, el Programa de las Naciones Unidas para el Desarrollo, Médicos Sin Fronteras, Migration Policiy Institue, Migration Data Portal.

La cantidad de remesas recibidas en los municipios hablan del papel tan importante que las comunidades migrantes tienen en el desarrollo de éstos; las matrículas consulares emitidas a jaliscienses en el exterior dan indicios de relaciones comerciales de la entidad federativa con otros países, a la par de que pueden mostrar cómo los cambios en política migratoria podrían llevar a un alza en la emigración. La información de jaliscienses en Estados Unidos refleja la historia migrante que tienen Jalisco y las redes migratorias en los Estados Unidos.

Por otro lado, la carencia de datos sobre las personas repatriadas pone en cuestión por qué estas personas regresan a un país que no los recuerda y no sabe qué hacer con ellos. Tal es la indiferencia que el gobierno tiene para este sector de la población migrante en retorno y deportados, que no se tiene la información de a qué municipio del país retornan, por ejemplo, en el caso de Jalisco: algunos de los municipios que conforman el Área Metropolitana de Guadalajara, en especial; Guadalajara, Zapopan y Tlaquepaque son de los principales municipios en recibir a personas migrantes en retorno y deportados, debido a los lazos familiares y la presencia de Call Centers, en donde gran parte de este sector de la población trabaja.

No obstante lo anterior, esto no se ve reflejado en ninguna estadística del gobierno local, y el conocimiento al respecto proviene principalmente de estudios académicos como, por ejemplo, los realizados por Mónica Jacobo en el Centro de Investigación y Docencia Económicas (CIDE) con el título: “Jóvenes migrantes en retorno: diagnóstico de trayectorias educativas y perspectivas de inserción laboral"; ${ }^{2}$ del informe "Los retos de comprobar que eres mexicano/a en tu propia tierra: El derecho a

${ }^{2}$ Para más información sobre migración de retorno, véase: https://bit.ly/3IlBwvH. 
la identidad de personas migrantes en retorno en Jalisco", 3 realizado por el Programa de Asuntos Migratorios del Instituto Tecnológico y de Estudios Superiores de Occidente (ITESO), o del presente ensayo.

El anuario analizado es un ejemplo muy claro del rol de los gobiernos locales y de cómo éstos comienzan a tener injerencia en el plano internacional, ${ }^{4}$ dado que poseen características idóneas (facultades y atribuciones) para articular demandas, defender sus intereses (en ocasiones no integrados al Estado central) y evaluar su lugar en las cuestiones internacionales, así como la forma en que éstas influyen en las dinámicas locales (Russell, 2006).

No queda la menor duda de que el anuario fue un trabajo reflexivo sobre el ejercicio de la paradiplomacia en términos muy técnicos. En el caso de Jalisco y como lo es para prácticamente todas las otras entidades federativas, se tiene mucho camino por recorrer en la generación de datos desagregados. A partir del trabajo realizado en este anuario, cambió la perspectiva de participación que las entidades federativas pueden y deben tener en la construcción de estadísticas de sus poblaciones migrantes; en primer lugar, porque a través de sus instituciones generan información que complementa los registros federales, y en segundo (el más importante), porque proveen de una visión más amplia acerca de la utilidad de la información para la implementación de políticas de atención y protección a las comunidades de migrantes. Los instrumentos para medir la migración no pueden ser entendidos sólo a nivel nacional, también deben entenderse localmente. En palabras de Sartori, (2012): "hemos empezado a correr sin haber aprendido a andar".

Cabe mencionar que el anuario se alinea a la Agenda 2030 y al Pacto Mundial Para una Migración Segura, Ordenada y Regular, ambos firmados y ratificados por el Gobierno Federal mexicano. La Agenda 2030 (A2030) surge a partir de la Cumbre de las Naciones Unidas sobre el Desarrollo Sostenible, en donde se aprobó el documento: “Transformar Nuestro Mundo: la Agenda 2030 para el Desarrollo Sostenible”, el cual incluye los 17 Objetivos del Desarrollo Sostenible (ODS) cuya meta es poner fin a la pobreza, luchar contra la desigualdad y la injusticia y hacer frente al cambio climático sin que nadie quede rezagado para el 2030 (Secretaría de Gobernación, 2016).

\footnotetext{
${ }^{3}$ Para más información sobre los retos de la doble nacionalidad, véase: https://clavigero.iteso.mx/category/edicion-6/.

${ }_{4} \mathrm{Al}$ revisar la nacionalidad de las personas que solicitaron refugio, se detecta a los países que están expulsando a su población, sea por crisis económica, política o social, y el aumento de las solicitudes de refugio en la entidad deja ver que Jalisco se está convirtiendo en un destino migratorio para personas sujetas a la protección internacional.
} 
Este se convirtió en el nuevo marco de desarrollo del Sistema de las Naciones Unidas (a nivel mundial y también para México), y está orientado a focalizar la cooperación y programación; a seguir abogando por y promoviendo la inclusión y la equidad en un marco de derechos, y construir más ciudadanía para las personas (Secretaría de Gobernación, 2016). La A2030 reconoce la importancia de los gobiernos y actores locales, tanto en términos generales como en el papel que tienen en la localización $^{5}$ de la A2030, lo cual resulta fundamental e idóneo en cuanto a paradiplomacia se refiere. Si bien el anuario no localiza como tal la A2030, sí es un ejercicio de alineación a ésta.

En concreto, los ODS abordan la migración internacional de forma subyacente y concomitante, porque aun cuando no está expresamente citada en alguno de sus objetivos como tal -al ser un tema transversal- está presente e inserta, en su generalidad, en cada uno de los objetivos (Secretaría de Gobernación, 2016). El ODS clave en migración es el número 8:

Promover el crecimiento económico sostenido, inclusivo y sostenible, el empleo pleno y productivo y el trabajo decente para todos. A través de Proteger los derechos laborales y promover un entorno de trabajo seguro y protegido para todos los trabajadores, incluidos los trabajadores migrantes, en particular las mujeres migrantes y las personas con empleos precarios (Secretaría de Gobernación, 2016).

Si bien este ODS es el único que tiene enunciación directa al tema migratorio, no se debe dejar de lado que la A2030 tiene una naturaleza transversal y que la migración internacional se encuentra nombrada en los 17 ODS (Secretaría de Gobernación, 2016). El trabajo Jalisco, un estado migrante. Anuario de migración de origen, destino, tránsito y retorno en Jalisco 2018, a la par d que el Reporte de Fondo de Apoyo a Migrantes, ambos realizados en el IJAMI por la Coordinación de Derechos Humanos y Desarrollo Integral, proporcionan información que propicia el cumplimiento de dicho ODS.

En más de una ocasión se ha dicho que la Organización de las Naciones Unidas y en general los Organismos Internacionales no funcionan como deberían y que las estrategias se alejan del contexto local y se quedan en el discurso. Es importante generar ejercicios en donde se exponga la importancia de que los gobiernos y actores locales

${ }^{5}$ En términos de la Agenda 2030, el concepto de localización es entendido como implementación; se utiliza dicha palabra debido a la naturaleza de la Agenda y el papel tan importante que representan los gobiernos locales en el cumplimiento de los Objetivos del Desarrollo Sostenible. 
consideren y trabajen en dichas estrategias de forma tal que éstas se adapten al contexto particular y la temática a abordar.

Por otro lado, el Pacto Mundial Para una Migración Segura, Ordenada y Regular tiene como antecedente la adopción de la Declaración de Nueva York en 2016. Fue a partir de ésta que los Estados miembros de las Naciones Unidas comenzaron a desarrollar este Pacto en cuya adopción México tuvo un papel muy importante, ya que, junto con Suiza, fungió como facilitador durante las negociaciones, buscando establecer un marco de cooperación internacional entre los actores estatales y no estatales relevantes del fenómeno migratorio.

El Pacto fue firmado en diciembre de 2018 en Marruecos y, aunque no es legalmente vinculante, considera algunos mecanismos de control para monitorear la implementación de los 23 objetivos que lo conforman por los Estados Parte. El anuario cumple y se alinea con el primer objetivo para la Migración Segura, Ordenada y Regular, el cual se refiere a la recopilación y utilización de datos precisos y desglosados como base para la generación de políticas basadas en pruebas e información (Relaciones Exteriores, 2018).

Como se puede observar este proyecto se vincula de diferentes formas y en distintos niveles a la carrera de Relaciones Internacionales; un trabajo que puede ser visto sólo como una recopilación de datos estadísticos, en las manos de un internacionalista termina mostrando la importancia de los gobiernos locales en fenómenos internacionales y su alineación, planificada o no, a las estrategias y pactos de los Organismos Internacionales.

ANÁLISIS DE LOS DESAFÍOS DE LA INSTITUCIONALIDAD LOCAL SOBRE LA MIGRACIÓN Y LA CARENCIA DE FUENTES DE INFORMACIÓN

Jalisco, un estado migrante. Anuario de migración de origen, destino, tránsito y retorno en Jalisco 2018 es un logro a nivel local, pues por primera vez un gobierno estatal se dio a la tarea de recopilar información estadística de temas migratorios. Vale la pena resaltar que Jalisco es el único estado a nivel nacional que cuenta con un documento con estadísticas sobre los cuatro flujos migratorios y las solicitudes de condición de refugio.

Sin embargo, aún queda camino por recorrer. Si bien el anuario fue un trabajo pertinente y expuso la situación migratoria que se vive en Jalisco, fue un proyecto no planificado, por lo que no contó con presupuesto asignado, lo cual, en términos de difusión y calidad del trabajo, es sumamente relevantes. Dentro del IJAMI era una persona 
la encargada de lograr que el anuario se realizara; esta persona desarrolló labores administrativas, de coordinación, investigación y recopilación de datos. La carencia de presupuesto implicó que el anuario fuera publicado solamente de forma virtual y que se imprimieran pocos ejemplares; finalmente la presentación del trabajo se hizo en un espacio pequeño, sin medios de comunicación, ni discurso del gobernador.

Adicionalmente se expone la falta de interés político por el tema migratorio, en dos niveles; el primero es meramente político: no se cuenta a quién no vota, en consecuencia, las personas solicitantes o refugiadas y personas inmigrantes con tarjetas de Residente Temporal o Permanente no figuran en el mapa de la población objetivo de los políticos, por lo que no se generan acciones de atención o se incluyen a estas poblaciones en los programas sociales; el segundo, es de carácter político-técnico y se ve reflejado en la carencia de fuentes para encontrar estadística desagregada a nivel estatal y municipal. Las entidades federativas no generan información de este tipo, todos los datos son generados por instituciones federales, de tal caso que el anuario es una recopilación de información obtenida de diferentes instituciones como el Instituto Nacional de Migración (INM), el Instituto de Mexicanos en el Exterior (IME), la Comisión Mexicana de Ayuda a Refugiados (COMAR) y el Banco de México (BANXICO). Esto expone la importancia de contar con áreas especializadas en los Institutos de geografía estatales o generar las vinculaciones pertinentes para producir información estadística.

Esta es una problemática que aqueja a los tomadores de decisiones en el plano local, estudiosos del tema migratorio, y a las organizaciones internacionales, pues es sumamente complicado encontrar información precisa que ayude a justificar la generación de políticas públicas, las reformas a leyes estatales de migración, la implementación de programas internacionales, incluso la apertura de oficinas de atención.

Debido a la situación migratoria en la que se encuentra el país, resulta sumamente importante abrir una oficina en dichos temas, que cuente con perfiles técnicos en la materia y esté vinculada con organizaciones internacionales, las cuales actualmente tienen mucho interés por conocer las dinámicas migratorias a nivel regional y estatal. Es también importante que esta oficina se encuentre sujeta a los cambios de administración, como lo estuvo el IJAMI: el caso de Jalisco es un claro ejemplo de retroceso en la atención a la población migrante, mientras que dicho instituto se contaba perfiles técnicos en materia migratoria, tenía un área especializada en casos y de atención directa a la población; se operaban dos programas sociales: el Fondo de Apoyo a Migrantes, de índole federal, y Familias Sin Fronteras, de carácter estatal, que contaba 
con un área dedicada a la elaboración de reportes de investigación sobre los programas y el propio presupuesto. Hoy Jalisco vive una realidad muy distinta.

Actualmente, la Dirección de Atención a Personas Migrantes del Gobierno del Estado carece de perfiles técnicos en temas migratorios. El Gobierno Federal derogó el Fondo de Apoyo a Migrantes, y el estatal Familias Sin Fronteras no tiene presupuesto, es una oficina de vinculación a otras dependencias y no una oficina de atención a las personas migrantes ni de generación de materiales de investigación. Si se hubiera dado seguimiento al proyecto del anuario, este año se estaría publicando el $4^{\text {to. }}$ anuario de migración del estado de Jalisco.

\section{COnClusiones}

La existencia de dependencias especializadas en el entendimiento y la atención de temas específicos resulta favorable para la generación de trabajos técnicos y vinculaciones en donde el nexo con la investigación sobre un tema particular, como el aquí analizado (la migración), generó información que me permitió entender los distintos flujos de personas migrantes desde una perspectiva inductiva. En otras palabras, si bien la migración es un fenómeno global, éste no puede ser entendido solamente desde un enfoque internacional; es necesario generar información desagregada que permita el análisis particular para originar diagnósticos locales, así como propuestas de políticas públicas e iniciativas de reforma de ley en el estado de Jalisco.

En este sentido, es importante resaltar que la perspectiva pro-persona en este tipo de actividades es fundamental para realizar un análisis integral de diferentes temas que influyen en los procesos migratorios, como las políticas públicas, los modelos de seguridad, el contexto de los países de origen, el desarrollo y la migración, siempre desde una perspectiva local.

Por otro lado, se evidencia la importancia de generar datos desagregados a niveles estatales, municipales y regionales para la mejor compresión de las problemáticas que aquejan a las poblaciones migrantes y no migrantes. Resulta fundamental reflexionar sobre los tiempos que corren y analizar la realidad que se vive en nuestra ciudad, en nuestro país, y conducir proyectos de investigación aplicada con el fin de aportar a la construcción del conocimiento y la defensa de los Derechos Humanos de las personas migrantes.

Finalmente, se subraya la importancia de conocer las instituciones, las responsabilidades y los alcances de los actores y gobiernos locales orientados a asuntos 
migratorios, y de evidenciar cómo lo internacional se construye desde lo local.

\section{REFERENCIAS}

Arenas, G. (2018). Paradiplomacia: Definiciones y trayectorias. Papel Político. Recuperado de https: / / bit.ly/3oUMEaV.

BAnCO DE MÉXICO (s/f). Sistema de Información Económica. 2020. Recuperado de https: / / bit.ly/3EXU6aP

BBC Mundo (s/f). Migraciones. 2020. Recuperado de https: / /bbc.in/31Z5zZd.

CNN EN ESPAÑOL (s/f). Migración. 2020. Recuperado de https: / / cnn.it/3ypCr9H.

Diccionario de La REAL ACADEMia ESPAÑOla (s/f). 2020. https: / / bit.ly/3EV4VdO.

DuRAND, J. (2012). El oficio de investigar. Métodos cualitativos y su aplicación empírica. Por los caminos de la investigación sobre migración internacional (pp. 47-75). UNAM-IIS, EL COLEF.

DuRAND, J. (2014). Coordenadas metodológicas. Dé cómo armar el rompecabezas. La etnografía y el trabajo de campo en las ciencias sociales (pp. 261-284). UNAM.

El País (s/f). Migración. Recuperado de https://bit.ly/3ysi1wP.

El Universal (s/f). Migración. 2020. Recuperado de https://bit.ly/3GIAS9U.

Excélsior (s/f). Migrantes. 2020. Recuperado de https: / / bit.ly/3pTwcap.

FM4 PASO LIBRE (s/f). Quiénes somos. 2020. Recuperado de https://bit.ly/3pQ7Rly.

IGLESIAS, M. (2020). Herramientas institucionales para promover la internacionalización de las ciudades mexicanas. Revista Tip (pp. 8-10). Recuperado de https: / / bit.ly/3yxIJUL.

InSTITUTO DE MEXICANOS EN EL EXTERIOR. Recuperado de https: / / bit.ly/3yoVJMu.

La Jornada (s/f). Migración. 2020. Recuperado de https: / / bit.ly/30pTD2e.

Le Monde (s/f). Immigration et diversité. 2020. Recuperado de https: / / bit.ly/3oQQUZ8.

Mexican Migration Project (s/f). 2020. Recuperado de https: / / bit.ly/3oS1tv4.

México reafirma su compromiso con el multilateralismo y con la gobernanza efectiva de la migración internacional. (2018). Relaciones Exteriores, Embajada en la Santa Sede. Recuperado de https: / / bit.ly/3dSsTdW.

MiLls, C. W. (1961). Sobre la artesanía intelectual. La imaginación sociología (pp. 206 236). Fondo de Cultura Económica.

New York Times (s/f). Inmmigration. 2020. Recuperado de https://nyti.ms/3GKUERW. 
Rodríguez Chávez, E., Cruz, S., María, L., y Martínez Caballero, G. (2012). Construyendo estadísticas: movilidad y migración internacional en México. Centro de Estudios Migratorios-Instituto Nacional de Migración-Tilde.

RuSSELL, R. (2006). El Estado-nación y los actores gubernamentales no centrales: su lugar y relación en el debate teórico dentro de la disciplina de las relaciones internacionales. La Paradiplomacia: las relaciones internacionales de los gobiernos locales (pp.17-41). Gobierno de Chiapas, Cámara de Diputados, Miguel Ángel Porrúa.

SARTORI, G. (2012). Cómo hacer ciencia política. Taurus.

SECRETARÍA DE GobernaCión (2016). La migración internacional en la Agenda 2030. Recuperado de https: / / bit.ly/3pPVgPu.

Secretaría de Planeación y Participación Ciudadana (2019). Plan Estatal de Gobernanza y Desarrollo de Jalisco $2018-2014$ (pp. 91-108). Gobierno de Jalisco. Recuperado de https: / / bit.ly/3dSK9Qg.

Secretaría de Planeación, Administración y Finanzas (2018). Manual de Organización y Procedimientos del Instituto Jalisciense para los Migrantes. Gobierno de Jalisco. Recuperado de https://bit.ly/3s6piBb.

SeCRETARÍA DE RELACIONES EXTERIORES (2018). México reafirma su compromiso con el multilateralismo y con la gobernanza efectiva de la migración internacional. Recuperado de https://bit.ly/3m7uYHw.

Secretaría General de Gobierno (2015). Periódico oficial El estado de Jalisco. Recuperado de https://bit.ly/3s0X4la.

SeCretaría General de Gobierno (2015). Decreto de creación del Instituto Jalisciense para los Migrantes. Secretaría General de Gobierno. Recuperado de https: / / bit.ly/3E8lstX.

Unidad DE POlítica Migratoria (2020). Boletines estadísticos. Recuperado de https: / / bit.ly/3IQdIQQ.

Viramontes MárqueZ, I. Y. (2018). Manual de Organización y Procedimientos de la Secretaría de Desarrollo e Integración Social. Tomo I: Gobierno del estado de JaliscoInstituto Jalisciense para Migrantes.

Viramontes Márquez, I. Y., CONTReras, A. eT AL. (2018). Jalisco, un estado migrante. Anuario de migración de origen, destino, tránsito y retorno en Jalisco 2018. Gobierno del Estado de Jalisco. 